Schöps, Miriam; Rumpf, Dietlinde

\title{
Universität mit Lernwerkstatt in Entwicklung. Von der Materialausleihe zur
}

\section{Hochschuldidaktik}

Kramer, Kathrin [Hrsg.]; Rumpf, Dietlinde [Hrsg.]; Schöps, Miriam [Hrsg.]; Winter, Stephanie [Hrsg.]: Hochschullernwerkstätten - Elemente von Hochschulentwicklung? Ein Rückblick auf 15 Jahre Hochschullernwerkstatt in Halle und andernorts. Bad Heilbrunn : Verlag Julius Klinkhardt 2020, S. 23-44. - (Lernen und Studieren in Lernwerkstätten)

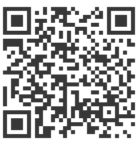

Quellenangabe/ Reference:

Schöps, Miriam; Rumpf, Dietlinde: Universität mit Lernwerkstatt in Entwicklung. Von der

Materialausleihe zur Hochschuldidaktik - In: Kramer, Kathrin [Hrsg.]; Rumpf, Dietlinde [Hrsg.]; Schöps, Miriam [Hrsg.]; Winter, Stephanie [Hrsg.]: Hochschullernwerkstätten - Elemente von

Hochschulentwicklung? Ein Rückblick auf 15 Jahre Hochschullernwerkstatt in Halle und andernorts.

Bad Heilbrunn : Verlag Julius Klinkhardt 2020, S. 23-44 - URN: urn:nbn:de:0111-pedocs-212001 - DOI:

10.25656/01:21200

https://nbn-resolving.org/urn:nbn:de:0111-pedocs-212001

https://doi.org/10.25656/01:21200

in Kooperation mit / in cooperation with:

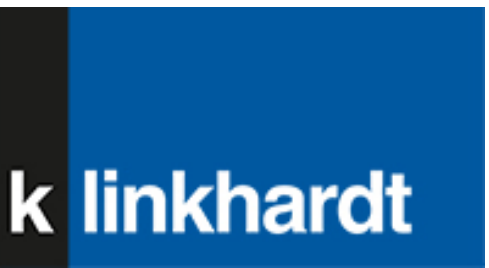

http://www.klinkhardt.de

\section{Nutzungsbedingungen}

Dieses Dokument steht unter folgender Creative Commons-Lizenz: http://creativecommons.org/licenses/by-nc-sa/4.0/deed.de - Sie dürfen das Werk bzw. den Inhalt unter folgenden Bedingungen vervielfältigen, verbreiten und öffentlich zugänglich machen sowie Abwandlungen und Bearbeitungen des Werkes bzw. Inhaltes anfertigen: Sie müssen den Namen des Autors/Rechteinhabers in der von ihm festgelegten Weise nennen. Dieses Werk bzw. der Inhalt darf nicht für kommerzielle Žwecke verwendet werden. Die neu entstandenen Werke bzw. Inhalte dürfen nur unter Verwendung von Lizenzbedingungen weitergegeben werden, die mit denen dieses Lizenzbedingungen weitergegeben werden,

Mit der Verwendung dieses Dokuments erkennen Sie die Nutzungsbedingungen an.

\section{Terms of use}

This document is published under following Creative Commons-License: http://creativecommons.org/licenses/by-nc-sa/4.0/deed.en - You may copy, distribute and transmit, adapt or exhibit the work in the public and alter, transform or change this work as long as you attribute the work in the manner specified by the author or licensor. You are not allowed to make commercial use of the work. If you alter, transform, or change this work in any way, you may distribute the resulting work only under this or a comparable license.

By using this particular document, you accept the above-stated conditions of use.

\section{Kontakt / Contact:}

\section{peDOCs}

DIPF | Leibniz-Institut für Bildungsforschung und Bildungsinformation Informationszentrum (IZ) Bildung

E-Mail: pedocs@dipf.de

Internet: www.pedocs.de

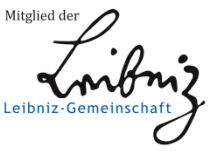




\section{Lernen und Studieren in Lernwerkstätten}

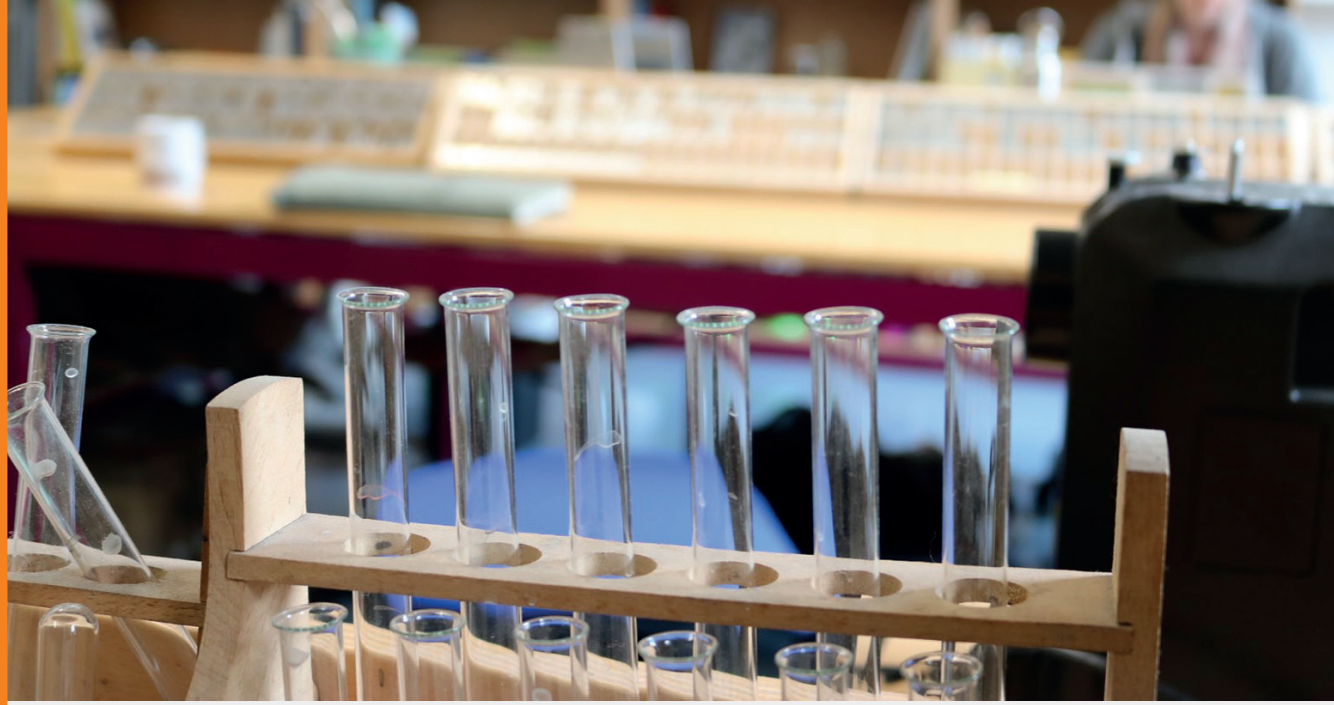

Kathrin Kramer / Dietlinde Rumpf / Miriam Schöps / Stephanie Winter (Hrsg.)

Hochschullernwerkstätten - Elemente von Hochschulentwicklung?

Ein Rückblick auf 15 Jahre Hochschullernwerkstatt in Halle und andernorts 


\section{Kramer / Rumpf / Schöps / Winter Hochschullernwerkstätten - Elemente von Hochschulentwicklung?}




\section{Lernen und Studieren in Lernwerkstätten}

Impulse für Theorie und Praxis

Herausgegeben von

Eva-Kristina Franz, Johannes Gunzenreiner, Barbara Müller-Naendrup, Hartmut Wedekind und Markus Peschel 


\section{Kathrin Kramer \\ Dietlinde Rumpf \\ Miriam Schöps \\ Stephanie Winter \\ (Hrsg.)}

\section{Hochschullernwerkstätten - Elemente von Hochschulentwicklung?}

Ein Rückblick auf 15 Jahre Hochschullernwerkstatt in Halle und andernorts 
Der vorliegende Band ist anlässlich einer Tagung des 15jährigen Bestehens der Hochschullernwerkstatt Erziehungswissenschaften in Halle im November 2020 entstanden.

Gefördert wurden die Tagung und der Band vom Zentrum für Lehrer *innenbildung, der Universitäts- und Landesbibliothek Sachsen-Anhalt sowie der Martin-Luther-Universität Halle-Wittenberg.

Dieser Titel wurde in das Programm des Verlages mittels eines Peer-Review-Verfahrens aufgenommen. Für weitere Informationen siehe www.klinkhardt.de.

Bibliografische Information der Deutschen Nationalbibliothek Die Deutsche Nationalbibliothek verzeichnet diese Publikation in der Deutschen Nationalbibliografie; detaillierte bibliografische Daten sind im Internet abrufbar über http://dnb.d-nb.de.

2020.k. (C) by Julius Klinkhardt.

Coverfoto: (C) Florian Johnke-Liese / Hochschullernwerkstatt.

Druck und Bindung: AZ Druck und Datentechnik, Kempten.

Printed in Germany 2020.

Gedruckt auf chlorfrei gebleichtem alterungsbeständigem Papier.

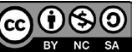

Die Publikation (mit Ausnahme aller Fotos, Grafiken und Abbildungen) ist veröffentlicht unter der Creative Commons-Lizenz: CC BY-NC-SA 4.0 International https://creativecommons.org/licenses/by-nc-sa/4.0/ 


\section{Inhalt}

Kathrin Kramer, Dietlinde Rumpf, Miriam Schöps und Stephanie Winter Einführung in den Band

\section{Teil 1: Historische Entwicklung}

Miriam Schöps und Dietlinde Rumpf

Universität mit Lernwerkstatt in Entwicklung -

Von der Materialausleihe zur Hochschuldidaktik

Hartmut Wenzel

Lernwerkstätten auch für die Sekundarstufen -

Zur Entwicklung der Hochschullernwerkstatt in Halle

Ulrike Stadler-Altmann, Susanne Schumacher, Enrico A. Emili,

Gerda Winkler und Elisabeth Dalla Torre

Hochschullernwerkstätten als Spielball der Bildungspolitik?

Die EduSpace Lernwerkstatt in der Südtiroler Lehrer*innenbildung zwischen nationalen und regionalen Bildungsinteressen

Hartmut Wedekind und Corinna Schmude

Von der Idee zum strukturell verankerten hochschuldidaktischen Prinzip -

Lernwerkstätten und Lernwerkstattarbeit im Studiengang „Erziehung und Bildung in der Kindheit" an der Alice Salomon Hochschule Berlin 68

\section{Teil 2: Wahrnehmung, Selbstverständnis, Einflüsse}

Dietlinde Rumpf und Corinna Schmude

NeHle - Internationales Netzwerk der Hochschullernwerkstätten -

Entwicklungsphasen einer Interessenvertretung und

eines gemeinsamen Begriffsverständnisses

Constantin Beyer und Florian Johnke-Liese

Hochschullernwerkstatt goes digital!

Alexandra und Michael Ritter

Drucken wie ,von gestern' in der Schule der Zukunft -

Die Schuldruckerei als Erfahrungsraum für Kinder und Studierende 
Livia Enders (geb. Makrinus) und Stephanie Winter

Studierende und Dozierende im Spannungsfeld zwischen

Theorie und Praxis - Die Hochschullernwerkstatt als Forschungsraum

John Marcus Sommer

Selbstwahrnehmung in der Hochschullernwerkstatt -

Wissenschaft zwischen Abstraktion und emotionalem Lernen

Lena Kliebe

Hochschullernwerkstatt als Raum und pädagogische Konzeption -

„Mein eigener Lernprozess“

Jerome Kampe

Geschichtsunterricht in der Lernwerkstatt!?

\section{Teil 3: Partizipation und Inklusion}

Melanie Schlag

Hochschullernwerkstatt schlägt Schlager

Kathrin Kramer

Nichtreformistische Reformen - Die Bedeutung von

Hochschullernwerkstätten auf dem Weg zu einer inklusiven Universität am Beispiel des Budgets für Arbeit

Johanna Ingenerf und Pascal Kurz

Menschenrechtsbasierte Bildung im internationalen Dialog -

Ein selbstorganisiertes Seminar mit Austausch

Ines Boban und Andreas Hinz

Hochschullernwerkstatt Halle als Möglichkeitsort -

Von WIRkstatt-Impulsen für inklusive Prozesse

Brigitte Kottmann und Alena Lensker (geb. Beckmann)

Die Lernwerkstatt und der Studiengang „Integrierte Sonderpädagogik“

an der Universität Bielefeld: Impulse, Synergien und Reflexionen

Sarah Dannemann, Tjark Neugebauer, Claudia Schomaker und Rolf Werning

Die LeibnizLernlandschaft: Diversität und Digitalisierung $\left(\mathrm{L}^{2} \mathrm{D}^{2}\right)$

gestalten - Konzeptionelle Gedanken für eine inklusive

Hochschullernwerkstatt an der Leibniz Universität Hannover 


\section{Teil 4: Reflexivität und Professionalisierung}

Kira Wybierek

Praxissemester in der Hochschullernwerkstatt -

Reflexion eines Lernprozesses

Eva Hoffart

„...da man lernt, eigene Gedanken und Ideen noch einmal zu vertiefen“ -

Theoretische Überlegungen und praktische Umsetzungen zum Reflektieren

von Lehramtsstudierenden

Edita Jung und Lena S. Kaiser

„Vielleicht romantisiere ich die Räume meiner Kindheit.“

Zugänge zu einer biographischen Reflexivität in

kindheitspädagogischen Hochschullernwerkstätten

Rolf-Torsten Kramer

Zum Problem der Professionalisierung im Lehramtsstudium und

zum Potenzial der Hochschullernwerkstatt

Mark Weißhaupt, Kathleen Panitz und Elke Hildebrandt

Die Inszenierung von „Theorie und Praxis“ sowie

„Neugier und Desinteresse“ bei der Professionalisierung

angehender Lehrpersonen in Hochschullernwerkstätten

Markus Peschel und Pascal Kihm

Hochschullernwerkstätten - Rollen, Rollenverständnisse und

Rollenaushandlungen

David Paulus, Patrick Gollub und Marcel Veber

Forschendes Lernen und Kasuistik: Überschneidungen und

Abgrenzungen bezogen auf Reflexivität in der

Hochschullernwerkstattarbeit

Pascal Kihm, Jenny Diener und Markus Peschel

Qualifizierungsprozesse und Qualifikationsarbeiten in

Hochschullernwerkstätten - Forschende Entwicklung einer

innovativen Didaktik 
10| Inhaltsverzeichnis

\section{Teil 5: Kooperation}

Georg Breidenstein, Sara Burkhardt, Thorid Rabe und Miriam Schöps

Zur Materialität des Lernens -

Anregungen aus einem interdisziplinären Forum

in der Hochschullernwerkstatt

Miriam Asmus, Kati Friebe, Mirjam Lewin und Kati Misselwitz

Entdeckendes Lernen und Digitale Medien - Ein Traumpaar .

Siglinde Spuller

Kooperation und Kooperatives Lernen als Prinzip

Hochschullernwerkstätten-adäquaten Lernens?

Eine konzeptionelle Verortung

Linda Balzer

Der Effekt selbstregulierenden und forschenden Lernens auf kooperative und individuelle Lernprozesse in der

Lernwerkstatt Religion Plural (LeRP)

Martin Lindner

Werkstattarbeit in der Biologiedidaktik -

Umstellung eines Praktikums auf Projektarbeit

Autor*innenbeschreibungen 


\section{Miriam Schöps und Dietlinde Rumpf}

\section{Universität mit Lernwerkstatt in Entwicklung - Von der Materialausleihe zur Hochschuldidaktik}

\section{Zusammenfassung}

Die Wurzeln der Hochschullernwerkstatt Erziehungswissenschaften an der Martin-Luther-Universität in Halle reichen bis in die 1990er Jahre zurück. Nach ortswechsel-vollen Anfängen hat sich die Hochschullernwerkstatt seit ihrer Entstehung in den letzten 15 Jahren zu einem festen Bestandteil der Philosophischen Fakultät III und zu einem wahrnehmbaren Element der Lehrer*innenbildung in Halle entwickelt. Trotz hoher Diversität der Ursprünge scheint das Verbindende all dieser Entwicklungen ein Innovationsanspruch an institutionalisiertes Lernen durch eine Veränderung der Lehrer*innenbildung zu sein. Die konkreten Aktivitäten, die in Hochschullernwerkstätten unter dieser Zielperspektive stattfinden, variieren deutlich. Sie folgen unterschiedlichen Orientierungen und sind geprägt durch äußere Bedingungen. Im Beitrag versuchen wir am Beispiel des Werdegangs der Hochschullernwerkstatt Erziehungswissenschaften die Entwicklung mit Blick auf zugrundeliegende Vorstellungen, interne und externe Einflussfaktoren und Wirkungen nachzuzeichnen. So soll der Frage nachgegangen werden, inwiefern Hochschullernwerkstätten auf die hochschulischen Entwicklungen einwirken (können) bzw. durch diese beeinflusst werden.

\section{Einleitung}

Im Jahr 2020 blicken wir auf 15 Jahre der Entwicklung „unserer“ Hochschullernwerkstatt Erziehungswissenschaften am heutigen Standort zurück und nehmen das zum Anlass, wichtige Etappen nachzuzeichnen, über Erreichtes, Nicht-Fortgeführtes und konzeptionelle Vorstellungen zu reflektieren und Zukunftsperspektiven zu schärfen. Dieses Innehalten gibt Gelegenheit, das Geschehen aus verschiedenen Perspektiven zu betrachten und im Verhältnis zu den Entwicklungen anderer Lernwerkstätten an Hochschulen zu sehen.

Im Rahmen der Rekonstruktion des Hauses 31 wurden auch Räume für eine Hochschullernwerkstatt geschaffen, die sich seit der Einweihung 2005 im universitär genutzten Teil der Franckeschen Stiftungen zu Halle direkt neben der 
erziehungswissenschaftlichen Bibliothek befinden. Ihr voran gingen mehrere Vorgängereinrichtungen, in denen Akteur*innen aus verschiedenen Fachrichtungen (Fachdidaktiken, Pädagogik, Förderpädagogik) und mit unterschiedlichen Zielstellungen wirkten. Die zu Grunde liegenden Motivationen und Orientierungen änderten sich mehrfach. Diese Entwicklung wird im Folgenden in einzelnen Phasen mit ihren unterschiedlichen Schwerpunkten vorgestellt.

\section{Entwicklungsphasen und zu Grunde liegende Orientierungen}

Während die Initialzündung für die Entstehung erster Lernwerkstatt-Räume an der Universität in Halle in der Schulpädagogik erfolgte, wurde zeitgleich am Institut für die Grundschullehrenden(aus)bildung in Köthen (PH) aus der Fachdidaktik heraus eine Lernwerkstattinitiative gestartet. Gründungen in weiteren Arbeitsbereichen schlossen sich an, Kooperationen waren allerdings bis zum Bezug der als Lernwerkstatt konzipierten Räumlichkeiten 10 Jahre später nicht gegeben. Tabelle 1 gibt einen Überblick über die Phasen der Entwicklung bzgl. der institutionellen Anbindungen, der personalen Verantwortlichkeiten und der Zielgruppen. Diese werden im Folgenden erläutert.

Tab. 1: Überblick der personell und konzeptionell zu unterscheidenden Entwicklungsphasen der Hochschullernwerkstatt Erziehungswissenschaften Halle

\begin{tabular}{|c|c|c|}
\hline Entwicklungsphase & Zeitraum & Fachbereich \\
\hline I a & 1994-1996 & $\begin{array}{l}\text { MLU Halle - Erziehungswissenschaften } \\
\text { Professur Schulpädagogik/Allgemeine Didaktik } \\
\text { Lehramt Sekundarstufe und Gymnasium }\end{array}$ \\
\hline Ib & 1993-1997 & $\begin{array}{l}\text { Ratke-Institut Köthen (PH) - Fachbereich Heimat- } \\
\text { und Sachunterricht } \\
\text { wissenschaftliche Mitarbeiterin (Werkstattleitung) } \\
\text { Lehramt Grundschule }\end{array}$ \\
\hline I C & 1998-2005 & $\begin{array}{l}\text { MLU Halle - Arbeitsbereich Deutsch } \\
\text { Professur Grundschuldidaktik/Deutsch } \\
\text { Lehramt Grundschule }\end{array}$ \\
\hline Id & 1997-2005 & $\begin{array}{l}\text { MLU Halle - Rehabilitationspädagogik } \\
\text { Professur Lernbehindertenpädagogik } \\
\text { Lehramt Sonder-/Förderschule und andere }\end{array}$ \\
\hline$\|$ & 2005-2011 & $\begin{array}{l}\text { MLU Halle - Philosophische Fakultät III } \\
\text { wiss. Mitarbeiterin (ohne Zeitkontingent) } \\
\text { Studierende aller Lehrämter }\end{array}$ \\
\hline
\end{tabular}




\begin{tabular}{lll}
\hline Entwicklungsphase & Zeitraum & Fachbereich \\
\hline III & MLU Halle - Philosophische Fakultät III \\
& & $\begin{array}{l}\text { wiss. Mitarbeiterin 50\% } \\
\text { alle Lehrämter und Erziehungswissenschaften, } \\
\\
\text { Schulen und außeruniversitäre Partner*innen }\end{array}$ \\
\hline IV & MLU Halle - Philosophische Fakultät III \\
& wiss. Mitarbeiterin 50\% \\
& alle Lehrämter und Erziehungswissenschaften, \\
& Lehrende und außeruniversitäre Partner*innen \\
\hline V & MLU Halle - Philosophische Fakultät III \\
& wiss. Mitarbeiterin 50\%, Projektmitarbeiterin 50\% \\
& alle Lehrämter, Erziehungswissenschaften, andere \\
& Fachbereiche und außeruniversitäre Partner*innen \\
\hline VI & derzeitige Bemühung: institutionell übergeordnete \\
& strukturelle Einbindung, Zuordnung einer \\
& Professur, kontinuierliche Verantwortlichkeit für \\
& Forschung und Organisation \\
\hline
\end{tabular}

\subsection{Phase I - Diverse Ursprünge (1993 bis 2005)}

Die vier Vorgänger-Institutionen der heutigen Hochschullernwerkstatt unterschieden sich im Hinblick auf ihre Zielstellungen, Schwerpunktsetzungen sowie Wegbereiter*innen deutlich.

\section{Ia Die (Meyersche) Lernwerkstatt der Pädagogik für den Sekundarschulbereich}

Mit seiner Berufung 1994 brachte Prof. Meinert Meyer (Professur für Allgemeine Didaktik/Schulforschung) in Zusammenarbeit mit seiner Mitarbeiterin Ingrid Kunze die Idee einer Lernwerkstatt in das Lehramtsstudium der Sekundarstufen ein. Er erhoffte sich, dass „die Studierenden in der Lernwerkstatt bei der Erstellung von Projekten aus ihren eigenen praktischen Problemen zur Theorie getrieben werden" (Kunze \& Meyer 1996). In diesen Projekten arbeiteten die Kollegen Reinhard Hörster und Hartmut Wenzel, der in seinem Beitrag in diesem Band darüber Auskunft gibt, mit.

Meyer wollte damit einen „Erprobungsraum für Lehramtsstudierende und Diplomer" (ebd.) etablieren, in dem sie, ausgehend von eigenen Erfahrungen selbstständigen, selbstgesteuerten Lernens, schließlich der Theorie bedürfen (vgl. ebd.). Dabei sollte sich die Rolle der Lernenden und Lehrenden verändern. Perspektivisch wurde eine Etablierung in den Fachbereichen angestrebt. Hier - an seiner ersten professoralen Wirkstätte - nahm bereits Meyers Grundidee Einfluss, die seine spätere Bildungsgangdidaktik prägte: „die Sinnkonstruktionen der Lernenden als Gestalter*innen ihrer eigenen Bildungsgänge ernst zu nehmen und Unterricht 
von dort her zu denken“ (Hericks et al. 2019, 142) und dies für die Allgemeine Didaktik und die Fachdidaktiken fruchtbar zu machen.

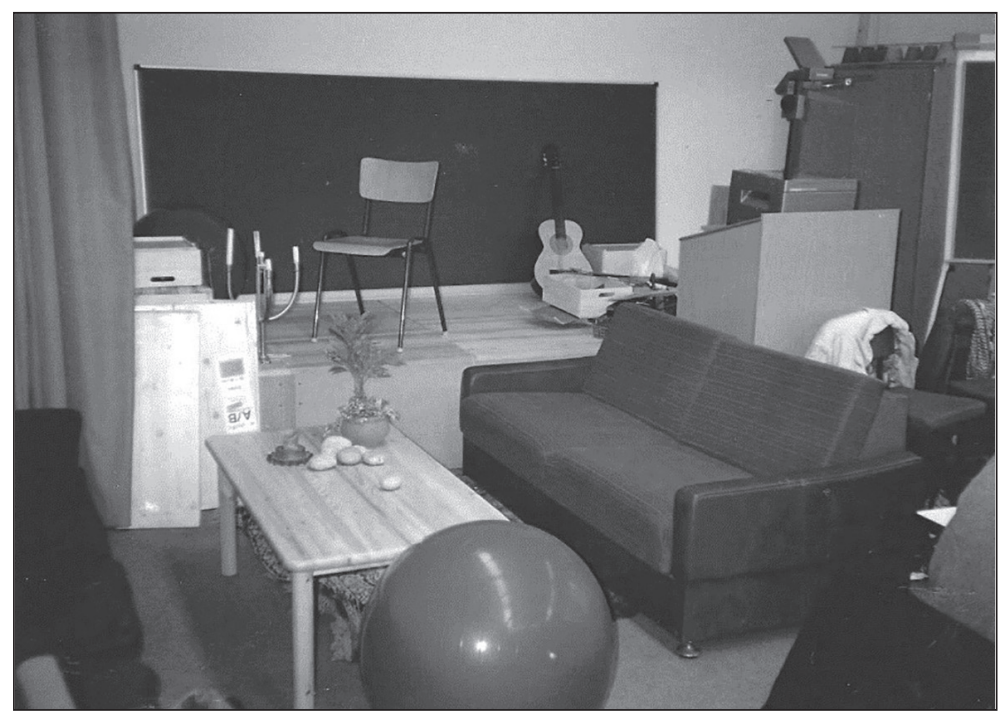

Abb. 1: Lernwerkstatt der Pädagogik für Sekundarstufenlehrämter ( $M$. Meyer)

Nicht nur die Einrichtung des Raumes am Standort Brandbergweg (Abb. 1), auch die inhaltliche Orientierung der Lehrveranstaltungen und außercurricularer Aktivitäten gingen auf Initiativen der Studierenden der Fächer Pädagogik und Soziologie zurück. So engagierte sich eine Gruppe für Bauspielplätze und organisierte die Erste Kinderstadt in Halle/Lettin mit Schüler*innen des Brennpunktviertels Heide-Nord. In einer Projektwoche setzten Kommiliton*innen museumspädagogische Angebote mit Schüler*innen im Grauen Hof in Aschersleben um. Eine weitere Gruppe konzipierte eine Zeitschrift für den sozialen Bereich. Die Professoren standen für Beratungen zur Verfügung und honorierten dieses Engagement durch „Projektscheine“. Durch die Unterstützung, die Meyer durch den Kanzler und den Dekan für diese Idee erfuhr, war es leicht, Räume zu etablieren (vgl. KunZE \& MeYer 1996) und Zeit seiner Tätigkeit in Halle und darüber hinaus zu nutzen.

\section{Ib Die Lernwerkstatt Heimat- und Sachunterricht im Ratke-Institut Köthen} Vor der Überführung der Pädagogischen Hochschule in die Universität 1997 gab es im Ratke-Institut Köthen, dem Standort für das Lehramtsstudium an Grundschulen in Sachsen-Anhalt, zudem bereits eine didaktische Lernwerkstatt.

Die dem Heimat- und Sachunterricht zugeordnete Werkstatt war thematisch natur- und umweltbezogen ausgerichtet und bot Möglichkeiten, naturwissen- 
schaftliche Themen handelnd, insbesondere durch Experimente zu erschließen. Die Konzeptionierung dieser Lernwerkstatt orientierte sich stark an den damaligen, als „Reform von unten“ aufgefassten Veränderungen der Grundschulen. Im Papier $Z u$ den Aufgaben der Lernwerkstätten an der Hochschule heißt es: „Die von Grundschullehrern geäußerte Kritik, daß die an den Grundschulen durchgeführten Reformen die Universitäten oft nicht erreichen, ist auch für uns überdenkenswert und sollte zu einer Öffnung und Erweiterung des Studienangebotes führen" (Schelenz 1993, 3). Mit den veränderten Anforderungen an Schule und Lehrkräfte (u. a. hinsichtlich methodischer Offenheit) wurde bereits die Notwendigkeit einer „Ablösung des zu stark rezeptiven und reproduktiven Lernens durch Formen des handelnden, experimentierenden, konstruktiven und kreativen Lernens" gesehen (ebd., 4). Die Anforderung einer „pädagogischen Professionalisierung des Grundschullehrers" wurde im Konzept festgehalten (ebd., 1) und als Zielstellung für die Lernwerkstatt präzisiert mit dem Zweck der Herausbildung pädagogischer Urteilsfähigkeit sowie einer kritischen Sensibilität beim Einsatz von Materialien (vgl. ebd., 3). Dies sollte durch die „Schaffung möglichst praxisnaher Bedingungen an der Hochschule“ erreicht werden, ,um den Studenten die Möglichkeit zu geben, theoretisch erworbenes Hochschulwissen unter grundschulmäßigen Voraussetzungen praktisch zu erproben [...]" (ebd.). Die Lernwerkstatt wurde einem "pädagogischen Labor" gleichgesetzt, aber auch als Ort des Erfahrungsaustausches im Sinne eines ,Bindeglieds zwischen Hochschule und Grundschule‘ verstanden - unter Bezugnahme auf die Gedanken der gerade aufkommenden Lernwerkstättenbewegung.

Die durch die Dozierenden im Bereich Heimat- und Sachunterricht gesetzte Aufgabenstellung dieser Lernwerkstatt war inhaltlich zunächst stark an den im Entstehen begriffenen Rahmenrichtlinien für den Heimat- und Sachunterricht und den gegebenen Bedingungen an der Pädagogischen Hochschule (Materialien, Fachbezug) orientiert, sollte sich aber „[i]m Rahmen der Weiterarbeit auf andere Sachverhalte zu fächerübergreifenden Themen“ als „wesentliches Kriterium" ausdehnen (ebd., 6).

Die Zusammenarbeit mit der 2. Phase der Lehrer*innenbildung wurde ebenso praktiziert wie der Erfahrungsaustausch mit den Lehrer*innen. Interessierten Schüler*innen war die Werkstatt im Rahmen außerunterrichtlicher Betätigung $\mathrm{zu}$ ausgeschriebenen Themen geöffnet. Jeden Monat wurde unter Leitung von Dr. Ute Schelenz (wissenschaftliche Mitarbeiterin des Arbeitsbereiches Heimatund Sachunterricht und Werkstattleiterin) mit Studierenden ein Thema in Stationen aufbereitet, um es für Grundschüler*innen anzubieten. 


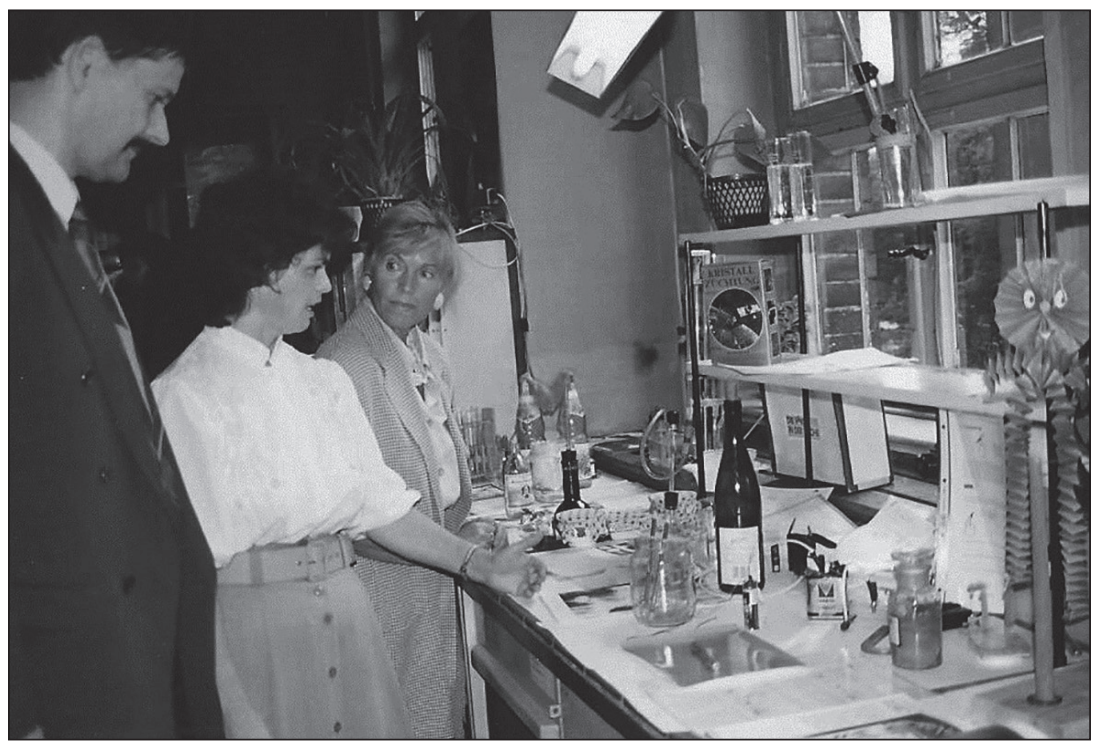

Abb. 2: Werkstattleiterin Ute Schelenz in der Lernwerkstatt mit dem späteren Bildungsminister des Landes, J.-H. Olbertz

Innerhalb der Hochschule wurde die Einbindung der Arbeit der Lernwerkstatt in die Lehrveranstaltungen der Fachdidaktiken (vgl. ebd., 2) unter anderem zu Schwerpunkten wie der Verknüpfung didaktischer Theorien mit konkreten „Lehrerhandlungen im Hinblick auf das Sammeln eigener Erfahrungen“ (ebd.) oder die Nutzung als Materialbörse in Vorbereitung auf die Schulpraktika formuliert.

\section{Ic, Id Die Druck- und Montessori-Werkstatträume}

Unter Leitung von Prof. Eva Maria Kohl wurde für das Fach Deutsch ein Werkstattraum mit den Schwerpunkten Drucken nach Freinet und szenisches Spiel eingerichtet. Anregungen wurden im Austausch mit Herbert Hagstedt (Leiter der Grundschulwerkstatt an der Universität Kassel) und aus der Konzeption der Lernwerkstatt für Studierende in Kassel gewonnen. Materialien und eine Handbibliothek für Freies Schreiben, Erzählen, Schrift und Schriftgestaltung sowie die Arbeit mit schreibenden Kindern, deren Schreiben als ästhetische Tätigkeit verstanden wurde, bildeten die Schwerpunkte auch in der didaktischen Ausrichtung der Lehrveranstaltungen. Regelmäßig wurden Projekte mit Kindern der Neumarkt-Grundschule durchgeführt, Vorläufer der später eingerichteten Schreibspielwiese, einem Zirkel schreibender Kinder aus halleschen Schulen und dem Saalekreis. Sie treffen sich bis heute in der Hochschullernwerkstatt und werden auch durch Studierende der Deutschdidaktik in ihrem Lernen begleitet. 


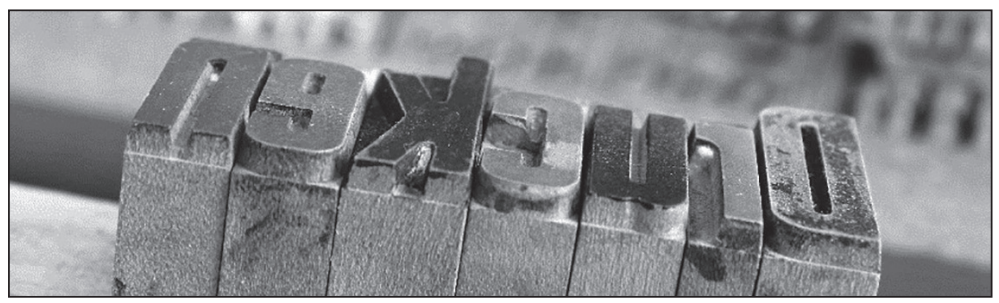

Abb. 3: Drucken als zentraler Arbeitsbereich der Werkstatt (@ N. Zeyn)

Für Studierende des Lehramtes an Förderschulen existierte eine Lernwerkstatt, die über einen umfangreichen Bestand an Montessori-Materialien verfügte - Materialien, die auch für Studierende aller Lehrämter in einzelnen Pädagogik-Lehrveranstaltungen herangezogen wurden. Diese Lernwerkstatt wurde von Ulrich Heimlich bei der Berufung auf die Professur für Lernbehindertenpädagogik 1994 ausgehandelt und zunächst, da dort Räume verfügbar waren und sie dem zuvor angeschafften Bestand an Montessori-Materialien Platz bot, im Haupthaus der damals neugegründeten Montessorischule direkt neben der Universität eingerichtet (vgl. KARGE \& HüBNER 2015). Dennoch war sie „nie dazu gedacht, mit Kindern drin zu arbeiten, sondern immer nur mit Studierenden" (ebd.). Es wurden exemplarisch Projekte erarbeitet, neue didaktische Ideen von Heimlich eingebracht, auch eine Freinet-Druckerei eingerichtet. Studierende gestalteten die ofenbeheizten Räume „liebevoll aus“ (ebd.). Nach dem Weggang des Professors zog diese Lernwerkstatt etwa drei Jahre später in die Universität zurück, da aber zeitweilig kein*e Mitarbeiter*in aus der Rehabilitationspädagogik die Expertise für die Montessori-Materialien besaß, wurden diese zunächst von Dr. Ursula Pilz (Grundschulpädagogik) mit genutzt, so dass eine erste Verbindung mit Lehrveranstaltungen der Allgemeinen Pädagogik erfolgte.

In diesen zwei Konzeptionen überwog der Aspekt, dass Hochschullernwerkstätten als Materialressourcen und Räume des handlungs- und materialbasierten Lernens fungierten, um so neue Lernformen zu erschließen.

\subsection{Phase II - Materialbasiertes Lernen \& Serviceorientierung (2005-2011)}

All diese Ansätze wirkten in die Hochschullernwerkstatt Erziehungswissenschaften hinein. Ausgangspunkt für die heutige Einrichtung, die diese bisherigen Werkstätten zusammenführte, war die räumliche Konzentration der Bildungswissenschaften in den sanierten Gebäuden der Franckeschen Stiftungen. Die Raumgestaltung der künftigen Hochschullernwerkstatt im Haus 31 wurde mit interessierten Dozierenden in Absprache mit den Kolleg*innen des zuständigen Ingenieurbüros Ziegemeier gemeinsam geplant. Die Einrichtung befand sich nun zentral und barrierefrei zugänglich im Hauptlehrgebäude des Bereichs Schulpä- 
dagogik, Rehabilitationspädagogik und der Grundschuldidaktiken der Lehrer*innenbildung Sachsen-Anhalt.

Am 8. Juni 2005 konnte die Lernwerkstatt der Fakultät Erziehungswissenschaften eröffnet werden, die auf $265 \mathrm{~m}^{2}$ drei Räume und ein Büro beherbergt. Davon waren zwei für Materialien und dazugehörende Fachliteratur mit entsprechenden Arbeitsplätzen (Abb. 4 unten) vorgesehen, im dritten Raum befinden sich eine Bühne (auch barrierefrei zu erreichen) und ein Klavier. Dieser ansonsten leere Raum kann bei Bedarf mit klappbaren Tischen und Stühlen ausgestattet werden. Alle Räume sind miteinander verbunden, sie werden kontinuierlich hinsichtlich der Barrierefreiheit und der wechselnden Nutzungsbedürfnisse weiterentwickelt.
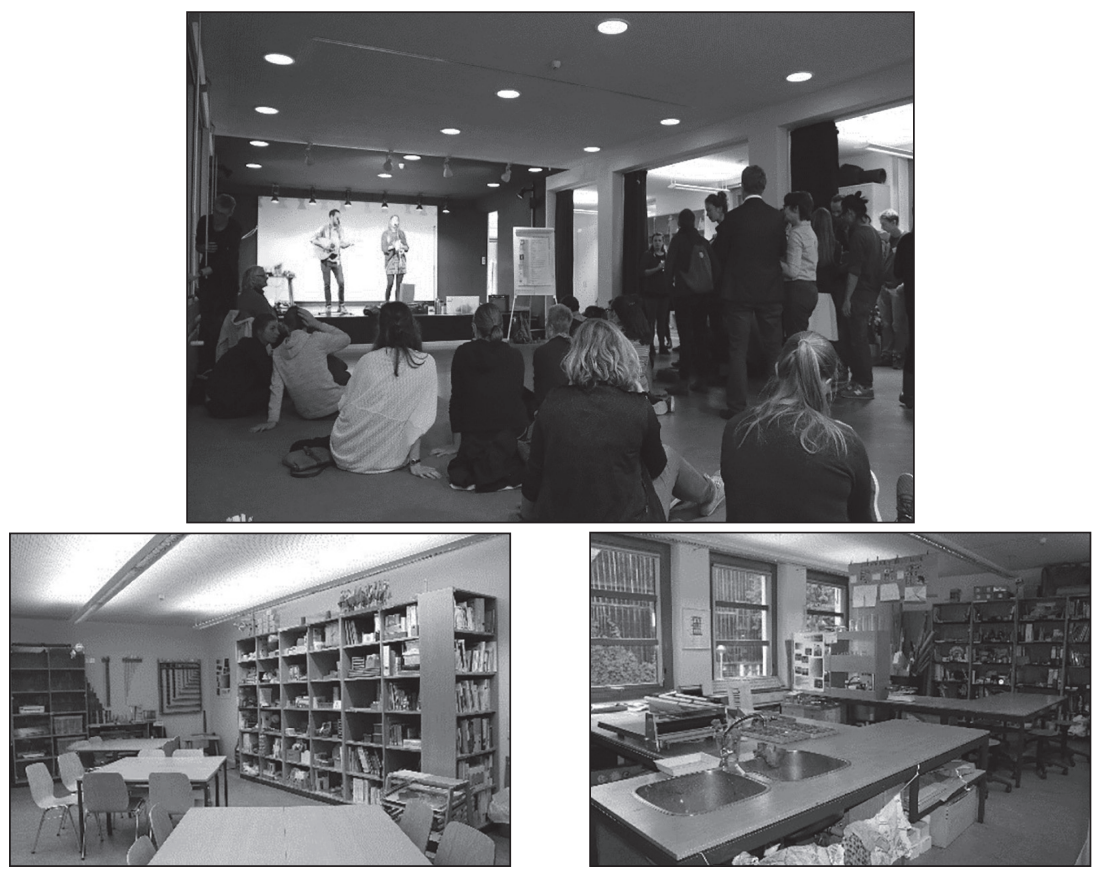

Abb. 4: Die Räume der Hochschullernwerkstatt (ㄷ N. Zeyn \& Team HSLW)

Die Hochschullernwerkstatt wird seitdem von der gesamten Fakultät verantwortet und versteht sich als Angebot für Studierende sowie Lehrende und Schulpraktiker*innen aller Schulstufen sowie der erziehungswissenschaftlichen BA- und MA-Studiengänge (vgl. Lw Ew 2015). Neben Lehrveranstaltungen wurde der Zugang zur Hochschullernwerkstatt während der Öffnungszeiten durch studentische Hilfskräfte mit insgesamt 20 Arbeitsstunden monatlich und einem Jahresetat von $500 €$ ermöglicht. Die Studierenden waren daran interessiert, Materialien 
und Literatur auszuleihen. Regelmäßig vor Ort arbeiteten allerdings nur wenige. Natürlich gab es Höhepunkte, wie die jährliche Weihnachtswerkstatt, die immer gut besucht waren.

Während Schwerpunkte der Vorläufereinrichtungen die Förderung selbstständigen Lernens und dadurch die Entfaltung eines ,Methodenrepertoires' einerseits, bzw. eine berufsorientierende Kompetenzentwicklung andererseits waren, ist für diese Phase vordergründig eine Material- und Serviceorientierung kennzeichnend. Der Mangel an zuständigem Personal (Weggang der Professoren, eine Mitarbeiterin ohne Stundendeputat) führten zur Einschränkung der inhaltlichen Entwicklung und aktiven Nutzung.

Obgleich in gemeinsamen Überlegungen aller Statusgruppen der Fakultät als sehr wichtig erachtet, Potenziale der Lernwerkstattidee innerhalb der Studiengänge zu etablieren, wurde dies kaum wirksam. Die Einbindung in Lehrveranstaltungen erfolgte vereinzelt und vorwiegend im Grundschulbereich. Die Studierenden konnten ohne Impulse durch die Dozierenden nicht für den Aufenthalt in der neu errichteten Werkstatt gewonnen werden. Möglicherweise unterstützte die Modularisierung mit ihren klaren Aufgaben und zeitlich anspruchsvollen Verpflichtungen diesen Trend.

\subsection{Phase III - Eigene Lernerfahrungen \& Lernbegleitung, Umgestaltung, Werkstattabende und Studierendenbeteiligung (2011-2015)}

Einen Übergang markierte das von Dietlinde Rumpf initiierte hochschulübergreifend ausgerichtete Kolloquium „Lernwerkstatt - Didaktische Chance oder Spielwiese im Lehramtsstudium?"“ am 15. Juni 2011 (Abb. 5). Es wurde im Rahmen des Seminars Konzeptionen von Lernwerkstätten vorbereitet. Die Studierenden diskutierten das Verständnis von ,ihrer' Lernwerkstatt und die hier erwünschten Möglichkeiten, die sie auf dem Kolloquium Kommiliton*innen und Dozierenden vorstellten. Auf diese Weise kamen Studierende aller Lehrämter und Lehrkräfte aus den verschiedenen Fachdidaktiken ins Gespräch. Es wurden zudem Ergebnisse eines Fragebogens, der an 6000 eingeschriebene Studierende gesandt und von 737 Lehramtsstudierenden beantwortet worden war, vorgestellt. Das wichtigste Ergebnis war, dass viele Studierende die Werkstatt kannten, aber kaum die intendierten Vorstellungen damit verbanden, sondern die Räume als Seminarräume mit den von den Dozierenden vorgeschlagenen Nutzungsmöglichkeiten wahrnahmen. Selten kannten Studierende der Sekundarstufenlehrämter die Lernwerkstatt, wobei sie - anders als die Studierenden der Lehrämter Grund- und Förderschule - kaum in dem Gebäude, in dem die Lernwerkstatt zu finden ist, Lehrveranstaltungen besuchen. Trotzdem gaben alle Studierenden an, dass solche Einrichtung in allen Schulformen sinnvoll wäre (vgl. RumpF \& SснӧPs 2013). 


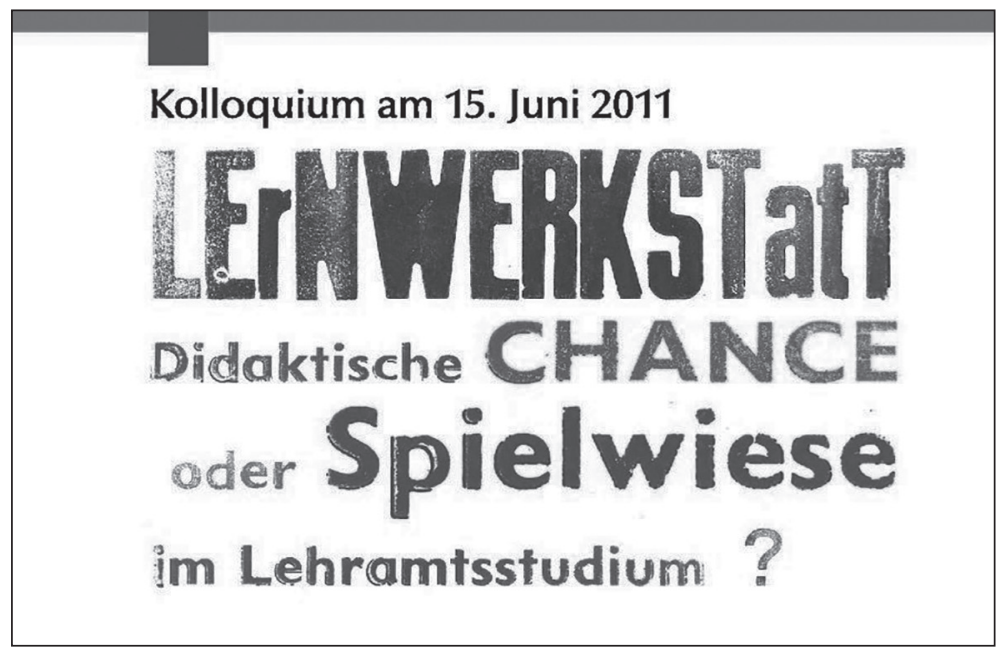

Abb. 5: Flyer zum Kolloquium 2011 ( A. Müller, Knick Design)

Überlegungen zu Aufgaben und Zielen einer Hochschulwerkstatt im Rahmen des Lehramtsstudiums schlossen die Tagung ab. Diese Ideen konnten aufgegriffen und verfolgt werden, da seit diesem Jahr mit Sponsorengeldern der Universität neben Sachmitteln eine Mitarbeiterin - erst stundenweise, später mit einer halben Stelle - gewonnen werden konnte. So wurde über die organisatorische Führung hinaus die inhaltliche Ausrichtung der Hochschullernwerkstatt zum Schwerpunkt der gemeinsamen Überlegungen.

Während die Ausgestaltung der Lernwerkstatt Erziehungswissenschaften bisher trotz einer fächerübergreifend gedachten Konzeptionierung inhaltlich nah an Unterrichtsfächern der Grundschule, Schulmaterialien und auf Rahmenrichtlinien orientiert war, wie sich in der Sortierung der Materialien widerspiegelte (vgl. Lw Ew 2009), wurde ab 2011 die Ausrichtung verstärkt auf erfahrungsorientiertes und reflexives Lernen gelenkt - in allen Altersklassen (auch im Sekundar- und Hochschulbereich), in offenen Formen, selbstverantwortet und entlang eigener zu entwickelnder Interessen (vgl. Schöps 2012). Dies schlug sich sowohl in den Angeboten als auch der Strukturierung des Materialrepertoires (Lernbereiche, Alltagmaterialien und spezifische Geräte) der Hochschullernwerkstatt nieder.

Eine grundsätzliche Entscheidung war die zur Materialanordnung, bezogen auf natur- und geisteswissenschaftliche Themen. Jeder Bereich sollte den Studierenden didaktische und methodische Anregungen bieten, die zur Bearbeitung für Kinder im Grundschul-, im Vorschulalter sowie für Schüler*innen der gymnasialen Stufe sinnvoll erschienen und allen Studierenden einen weiten Horizont im Umgang mit einem Thema eröffnen, ausgehend eben nicht von einer Fächerlogik. 
Orientierung fand sich in Klafkis epochaltypischen Schlüsselproblemen (KLAFKI \& Braun 2007, 165, 168), ergänzt durch Vorstellungen aus Toni Bоотн' Inclusive curricula for all (Воотн \& Ainscow 2011, 15).

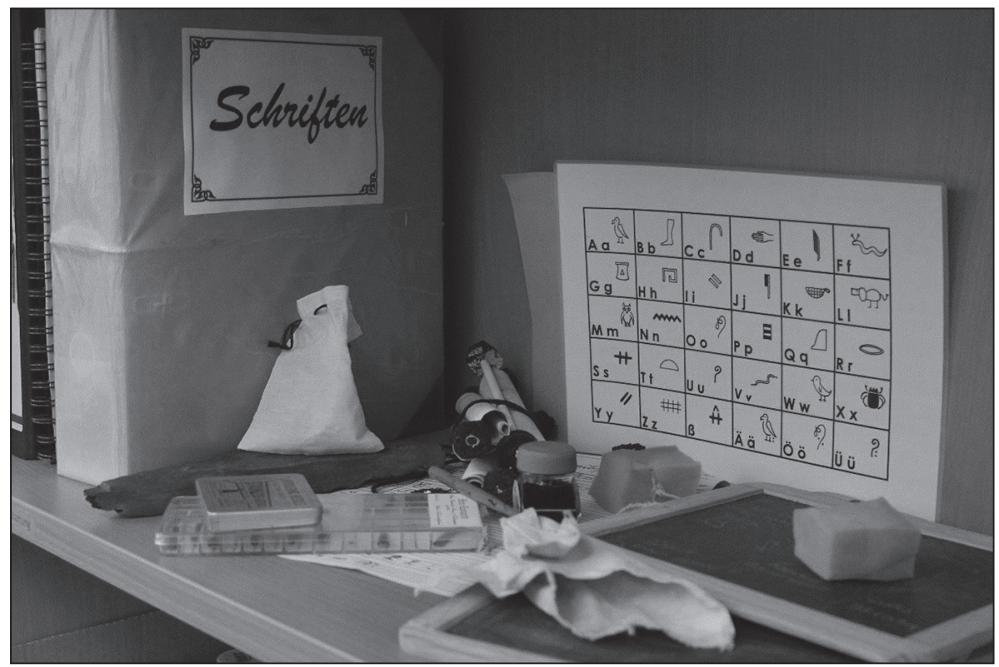

Abb. 6: Regal zur Schrift ( N. Zeyn)

Neben Überblickswissen zu Lernwerkstattarbeit, zur Öffnung des Unterrichts und zu Konzeptionen, die mit Material arbeiten, stehen Bücher und Handreichungen gleich neben den Objekten in den Regalen, auf die sie sich beziehen. In Kooperation mit der erziehungswissenschaftlichen Zweigbibliothek der Universität werden die Publikationen angeschafft und im OPAC verbucht. (Abb. 6)

Ein weiterer Ansatz, die Studierenden zu erreichen, war die systematische Information über die Möglichkeiten, welche die Hochschullernwerkstatt bot - in Form von Werkstattblättern ${ }^{1}$ (was sich wenig bewährte), auf der Homepage und der Studienplattform, mit Plakaten, Flyern und schließlich auf social media-Plattformen ${ }^{2}$. Auch die Raumbuchungsmöglichkeit wurde für Studierendengruppen eröffnet, so dass sowohl die vorhandenen Materialien als auch die Räume selbst den Studierenden auch außerhalb der Öffnungszeiten verfügbar wurden.

Ab 2013 wurden - bald in Kooperation mit interessierten Studierenden - Werkstattabende veranstaltet: für alle Interessierten offene und kostenfreie Workshops oder Diskussionsrunden zu Themen, die im Studium kaum Eingang fanden. Do-

1 Sснӧрs, Miriam \& Röwer, Susann (2014): Werkstattblatt 1: Offene Themenabende und Seminare zum entdeckenden Lernen - Die Lernwerkstatt Erziehungswissenschaften stellt sich vor. Frühjahr/ Sommer 2014, MLU Halle

2 Facebook-Seite: https:/www.facebook.com/LernwerkstattEWS/ 
zierende, Studierende oder Expert*innen gestalteten die Angebote, die viel Zulauf fanden und bald Semesterprogramme füllten (vgl. Abb. 7).

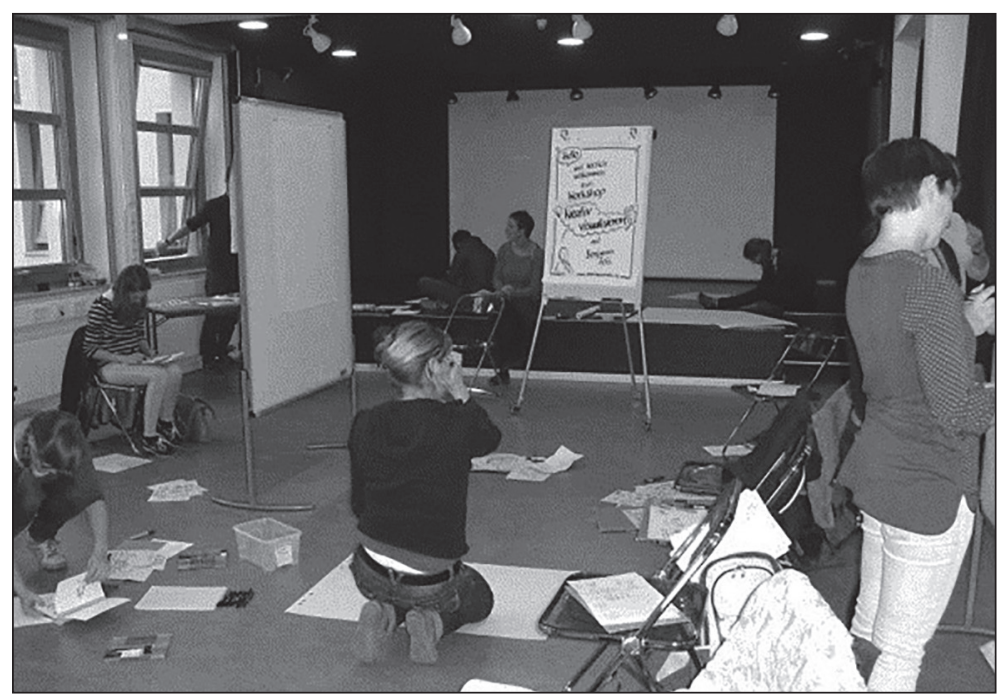

Abb. 7: Werkstattabend „Visualisieren“ 2014 (ㄷ M. Schöps)

Die Kooperationen mit Schulen wurden in Austauschrunden, die regionale Akteur*innen von Schul-Werkstätten zusammenbrachten - Werkstattgespräche ${ }^{3}$ von 2011-13 - sowie durch die Thematisierung in Seminaren (zu Konzeptentwicklungen u.ä.) vertieft.

Die intensive Einbindung in die beiden Netzwerke der im deutschsprachigen Raum existierenden (Hochschul)Lernwerkstätten (VeLW (ehem. Verein), NeHle und die Verbindung der Lernwerkstatt-Praktiker*innen) sowie die Teilnahme an den jährlichen Tagungen beider Gruppen - teilweise mit Studierenden - gaben viele Impulse für Lehre und Organisationsentwicklung. Damit einher ging eine zunehmende Forschungs- und Publikationsorientierung, welche unter anderem die Auseinandersetzung mit Fragen nach Professionalisierung und inklusionsorientierter Entwicklung der Hochschullernwerkstatt und der Universität mit sich brachte (vgl. Schöps 2016).

Aus Begegnungen auf den Europäischen Lernwerkstättentagungen (Praktiker*innentagung) in Linz 2013 und Blossin 2014 erwuchs eine Kooperation zwischen Miriam Schöps und Karen Graf (LIFE e.V Berlin), wodurch ein Seminarkonzept entwickelt, modifiziert und etabliert wurde. Es stellte die Selbsterfahrung

3 Lernwerkstatt für Schulen:

https://www.philfak3.uni-halle.de/institut/hochschullernwerkstatt/links/informationenschulen/ 
entdeckend-forschenden Lernens und des Begleitet-Werdens ins Zentrum. Diese Fokussierung auf eigene Lernerfahrungen der Selbstregulation und Frageorientierung, durch konkrete Impulse der (Selbst-)Reflexion zugänglich gemacht, bildete den Schwerpunkt der Lehraktivitäten in dieser Etappe.

Davon ausgehend wurde die Lernbegleitung als veränderte Lehrendenrolle Thema pädagogischer Reflexion und später in mit engagierten Studierenden und Hilfskräften gemeinsam entwickelten Projekten (im außerschulischen Kontext) erprobt (vgl. Schöps 2019, 2015; Rumpf \& Schöps 2017). Ziel dieser Projekte war es, einen leistungsdruckentlasteten Rahmen zu bieten, offene Lernformen in einer von Anerkennung geprägten Grundhaltung gemeinsam auszuprobieren, sich beraten und gegenseitig beobachten zu können. Dazu wurden regelmäßige Kooperationen mit einem Gymnasium und einem Träger der Jugendhilfe, welche durch die Werkstattgespräche entstanden waren, verfestigt. Diese konnten nach 2019 auf Grund fehlender Ressourcen nicht fortgeführt werden.

Die Öffnung innerhalb der Universität bildete sich zunehmend in Form von Lehrkooperationen insbesondere mit den Fachdidaktiken im Sekundarbereich (Deutsch, Spanisch, Geschichte, Mathematik) aus, welche oft auf Studierendeninitiativen zurückgingen. Hier richtete sich der Fokus jedoch vornehmlich auf materialgeleitetes Lernen im Fachunterricht.

Die wiederkehrende Frage nach Professionalisierungspotenzialen wurde im Rahmen der Tagung zum 10. Jubiläum der Lernwerkstatt Erziehungswissenschaften mit dem Thema Lernwerkstätten und die Professionalität von Lehrer*innen in einer zukünftigen Schule aufgegriffen. In Form von einer Filmdiskussion, Vorträgen und Workshops für Studierende, Dozierende und Lehrer*innen - gerahmt von Beiträgen des Studierendentheaters und Bandkonzerten - wurde die Frage an die interessierte Gemeinschaft gestellt: Welche professionellen Kompetenzen müssen und können - wie? - in unserer Hochschullernwerkstatt ausgebildet werden? (vgl. Sснӧps \& RumpF 2015).

Die Bereiche, die uns dabei interessierten, waren: Professionalisierung für Lernwerkstattarbeit (Praxisrelevanz), Professionalisierung in Lernwerkstätten (Anknüpfungspunkte für curricular verankerte Lerninhalte etc. (vgl. KRAMER, R. in diesem Band) sowie Professionalität und Professionalisierung von Dozierenden für die Werkstattarbeit (Entwicklung lernbegleitungsprofessioneller Kompetenzen).

Die Perspektive auf Hochschullernwerkstatt änderte und vertiefte sich im Verlauf dieser Entwicklungsphase vom diffusen, aber offenen „Ort für Studienkultur“ (Sснӧps 2012) zur Wahrnehmung der Hochschullernwerkstatt als „eine modellhafte Lernumgebung, die Studierenden forschendes Lernen ermöglicht und vielfältige Zugänge zur reflektierten Theoriebildung bietet" (vgl. Schöps \& RuMPF 2015). Im Rückblick auf diese Etappe können die konzeptionellen Zielstellungen der Hochschullernwerkstatt damit bestimmt werden: Einen Raum für Selbsterfahrung sowie reflektiertes pädagogisches Probehandeln schulstufenübergreifend 
zu etablieren und die Reflexion über veränderte Lernkultur und die veränderte Rolle der Pädagog*innen - auch in der Hochschule - zu befördern (vgl. ebd.). Persönlichkeitsbildung sowie Berufsorientierung standen sich dabei stellenweise auch widerstreitend gegenüber.

\subsection{Phase IV - Kooperation, Inklusion und Kasuistik (ab 2015)}

In der Folge der Tagung konnten für die Weiterentwicklung der Hochschullernwerkstatt mehrere Studierende gewonnen und mit Hilfskraftmitteln über den Hochschulpakt finanziert werden. Ein wachsendes studentisches Team wurde bei der konzeptionellen und inhaltlichen Weiterentwicklung immer stärker eingebunden. Anschließend an oben beschriebene Projektprofilierung wurden, gemeinsam mit diesen studentischen Hilfskräften, Projekte mit Studierenden und Jugendlichen kooperierender Einrichtungen zur Gestaltung, Begleitung und reflexiven Betrachtung offenen entdeckenden Lernens entlang einer eigenen Fragestellung entwickelt. Ab 2016 konnten Studierende diese auch im Rahmen eines Praktikumsmoduls belegen und wurden so anhand eigener Beobachtungsdaten aus der Lernwerkstatt weiter in die kasuistische Arbeit eingeführt (vgl. LeweK 2019). Hier verlagerte sich der Schwerpunkt: von eigenen Lernerfahrungen auf die pädagogische Gestaltung von Projekten und die Erprobung der Lehrendenrolle als Lernbegleitung. Die Dokumentation und Analyse von Fallbeispielen dieser ,eigenen Praxis ergänzte schließlich die begleiteten Reflexionen (vgl. Schöps 2019, 110).

Die Organisationsentwicklung betreffend wurde teamintern die Entwicklung der Hochschullernwerkstatt als inklusiver (Lern)Ort bedeutsamer. Es wurde ein Modus entwickelt, Schulpraktikant*innen einer freien Schule (Schüler*innen mit Förderbedarf und einem spezifischen Praktikumsformat) über lange Zeiträume in die organisatorischen Arbeiten der Hochschullernwerkstatt einzubinden und so im Team wie auch bei Nutzer*innen Austausch und Reflexionen über Lernund Zugangsmöglichkeiten zu Bildungssystemen unter anderen Voraussetzungen anzuregen.

Ziel dieser Phasen war es, Potenziale der Werkstattarbeit in viele Richtungen auszuloten. Dazu hatten wir einerseits Seminare für Studierende aller Lehrämter innerhalb des Curriculums mit verschiedenen Schwerpunkten angeboten, andererseits alle Initiativen gefördert, die spannende Ideen in der Lernwerkstatt umsetzen wollten. Daraus ergaben sich durchaus diffuse Aktionen, die nicht immer zielgerichtet auf das, was wir unter Lernwerkstattarbeit verstehen, zusteuerten. Häufig waren einfach der gemütliche Raum und Getränke der Anziehungspunkt. Allerdings zeigte sich oft erst im Nachhinein, welche Vernetzungen und Kooperationen und daraus erwachsende Potenziale sich ergaben. Studierende unterschiedlicher Lehrämter, Dozierende, Referendar*innen und verschiedene Statusgruppen zusammen zu bringen, ist für uns ein wichtiger Aspekt in der Lernwerkstattarbeit. 
Im Rahmen des KALEI-Projektes, welches Heterogenitätssensibilisierung durch Kasuistik im Lehramtsstudium fokussierte, bereicherten neue theoretische Perspektiven die Arbeit in der Hochschullernwerkstatt. Fallbasiertes Arbeiten wurde neben Lernmaterialanalysen zentraler Gegenstand von kooperativ entwickelten Lehransätzen (bspw. zur Mathematik-Didaktik in Verbindung mit Integrationspädagogik mit Prof.in Richter und Prof. Veber) oder der Evaluation ganzer Lehrveranstaltungen mit rekonstruktiv-kasuistischem Lehransatz (vgl. GRUMmT et al. 2019a, 2019b). Durch den projektgebundenen personalen Aufwuchs (eine 50\%-Stelle für 2,8 Jahre) konnte der weitere konzeptionelle Ausbau der Hochschullernwerkstatt (vgl. Sснӧрs 2017) intensiviert werden.

\subsection{Phase V: Hochschuldidaktik, Vernetzung, Demokratie \& Digitalisierung}

Die Einbindung in das universitäre Netzwerk der lehrer*innenbildenden Bereiche aller Fakultäten im Rahmen des KALEI-Projektes führte zur Weiterentwicklung von Austausch- und Kooperationsformaten und zu einer gesteigerten Wahrnehmung der Hochschullernwerkstatt in der Universität. In das Forum Zur Materialität des Lernens (BREIDENSTEIN et al. in diesem Band) bspw., welches den Austausch von Studierenden, Fachdidaktiker*innen, Pädagog*innen und Interessierten zu Perspektiven auf Unterrichtsmaterialien und deren Einbindung ermöglicht, bringen sich zahlreiche Fachbereiche ein.

Impulse und zeitliche Ressourcen durch den personalen Aufwuchs ermöglichten verstärkte Einbindungen in lokale und regionale Netzwerke (open-lab-net Halle u. a.). Dadurch halten aktuelle Diskurse verstärkt Einzug in die Themen und Angebote der Hochschullernwerkstatt (bspw. Semesterthemen: Medien und Digitalisierung, Kommunikation, Sexualität und Gesellschaft, Nachhaltigkeit etc.) und erleichtern die Kooperation mit externen Expert*innen. Diese Vernetzungen ermöglichten auch die Weiterführung der Praktikumsverhältnisse der zwei Mitarbeiter*innen mit Förderbedarf nach Bundesteilhabegesetz über deren Schulzeit hinaus und die Schaffung einer Bundesfreiwilligendienst-Stelle. Diese wurde von einem Mitarbeiter, der aus Syrien geflüchtet war, besetzt und ermöglichte so interkulturellen Austausch sowie (Sprach- und andere) Lerngelegenheiten auf niedrigschwelliger, informeller Ebene. In einem der Fälle gelang durch intensives Engagement, unterstützt durch die gerade berufene Professorin für Inklusion und Exklusion in Bildung, Erziehung und Sozialisation und zuallererst durch die Mitarbeiterin selbst, die Schaffung einer Arbeitsstelle (vgl. Schlag und Kramer, $\mathrm{K}$. in diesem Band). Sie nimmt nun eine wichtige und fakultätsweit be- und anerkannte Position in der Hochschullernwerkstatt ein. Diese Entwicklungen wirken auf Universitätsstrukturen zurück. Dabei wird aber auch deutlich, dass der hohe organisatorische Aufwand, zum Teil jahrelange Entscheidungswege einerseits sowie die kontinuierliche Entwicklung von Lehrangeboten und -kooperationen 
andererseits die Absicherung einer langfristigen Stelle einer*s wissenschaftlichen Mitarbeiter*in erforderlich macht.

Mit zunehmender ,Raumeroberung' durch Studierende (auch durch die Einrichtung einer Kaffee- und Wohlfühlecke) und bisher nicht involvierte Dozierende stieg die Nachfrage nach Nutzungszeiten. Mit multipleren Aktivitäten wird die Bestimmung des Bezuges „Ist das Lernwerkstatt?“ und „Welches Nutzungsanliegen ist eher berechtigt?"“ schwieriger. Die Ausschärfung des Konzeptes wurde nötig, da dies unweigerlich mit Veränderungen gängiger Praxis einhergeht. In Formaten wie dem Tag der Lehre 2018 erarbeiteten sich Studierende und Dozierende der gesamten Fakultät Entwicklungsmöglichkeiten der Hochschullernwerkstatt. Dabei wurden Perspektiven entwickelt, die die Etablierung einer supervisorischen Fallwerkstatt für Studierende, hochschuldidaktische Konzepte und die Umgestaltung der Räume betreffen.

Wiederkehrend ist auch die Diskussion um die curriculare Verankerung der Hochschullernwerkstatt: Während einem studienorganisatorisch honorierten Angebot (durch Zertifikate oder Leistungspunkte) einerseits das Potenzial unterstellt wurde, dass mehr Studierende, lernwerkstattartigem' Lernen in der obligatorischen Lehre begegnen, wie an der Universität Erfurt (vgl. GodAu et al. 2018), andererseits wird der Hochschullernwerkstatt „ein besonderes Potenzial als Bildungs- und Reflexionsraum“ zugedacht, gerade „weil sie außerhalb der curricularen Modulstruktur platziert ist (Kramer, R. in diesem Band). Diese ,Freiheit' als Kontext selbstbestimmten Lernens steht anschließend im Zentrum der Gestaltung von Angeboten und Aktivitäten.

Die dem Tag der Lehre folgende Umgestaltung der Räume unter Einbezug der Studierenden bewirkte eine gemütlichere Atmosphäre und so die vermehrte Nutzung der Hochschullernwerkstatt als Arbeits- und sozialem Ort (vgl. Abb. 8).

Dieser Umgestaltung zuträglich war auch die im Kontext der Digitalisierung der hochschulischen Lehre beantragte Einrichtung einer Medienwerkstatt (HsLw 2018). Sie wurde schrittweise durch die Anschaffung neuer Geräte und digitaler Werkzeuge (Plotter, 3-D-Drucker, PC-Arbeitsplatz etc.), die Einstellung kompetenter Hilfskräfte zur Betreuung von Lernangeboten, einer Beratungsstunde für Dozierende und Studierende und die Arbeitsplatzeinrichtung umgesetzt. Sie erforderte eine Umgestaltung des Freiarbeitsraumes und eine Reduktion der z.T. alten didaktischen Materialien (siehe hierzu auch BEYER \& JoHNKe-Liese in diesem Buch). Die Nutzung dieser digitalen Medien stellten Beyer und Johnke-Liese in einem Workshop auf der Jahrestagung in Wien 2020 vor. Im Rahmen der in Halle ausgerichteten Europäischen Lernwerkstättentagung im Mai 2018 entdeckendes lernen - materialbasiert und medienunterstützt waren sie zentraler Gegenstand der Werkstattarbeit (vgl. Asmus et al. in diesem Band). 


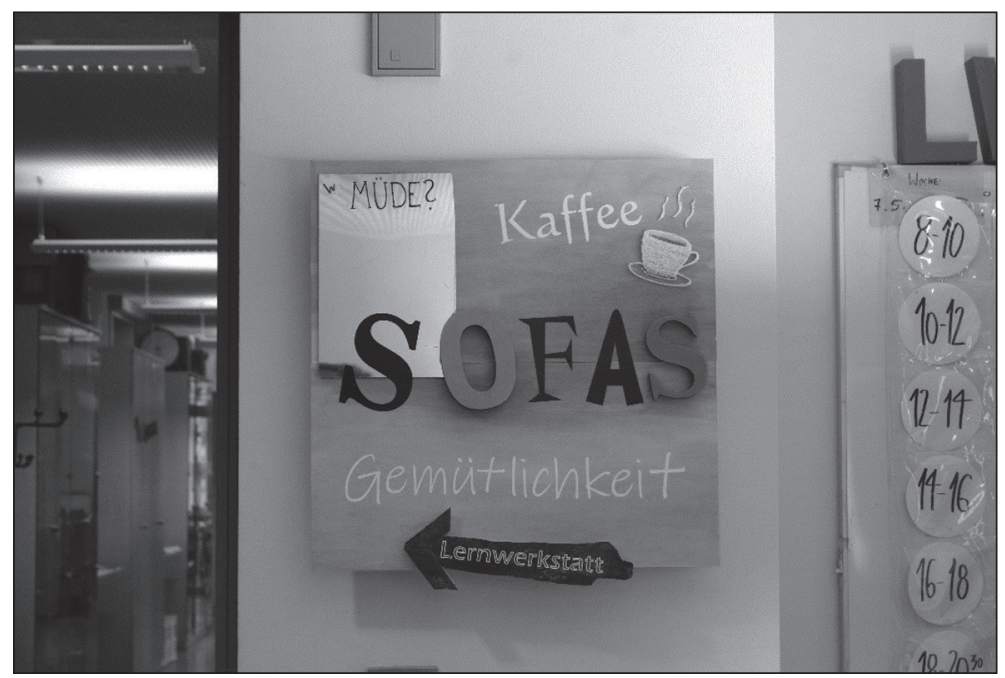

Abb. 8: Hochschullernwerkstatt als gemütlicher Ort (@ K. Kramer/Team HSLW)

Zudem nutzen zunehmend mehr Dozierende, vor allem Didaktiker*innen des Sekundarschulbereiches, die Hochschullernwerkstatt als Raum für die Lehre. Hieraus ergaben sich individuelle Beratungen und bisher zwei Treffen zum „hochschuldidaktischen Austausch" über Lehrkonzepte und organisatorische Fragen. Dies konnte allerdings nicht kontinuierlich fortgesetzt werden und bedarf passenderer Veranstaltungskonzepte. Ein Interesse der Mitarbeiter*innen der Hochschullernwerkstatt ist es weiterhin, die Dozierendenrolle stärker in den Blick zu nehmen (vgl. Kramer et al. 2019).

Die in den letzten Jahren begonnene außeruniversitäre Vernetzung im Wissenschafts- und Bildungsbereich durch Tagungsteilnahmen und einzelne Kooperationen wird in dieser Zeit intensiviert. Sie mündet in der Gründungsmitgliedschaft im Internationalen Netzwerk der Hochschullernwerkstätten e.V. NeHle (2019) einerseits und dem weiteren Ausbau und der Verstetigung zahlreicher Kooperationen mit Vereinen und regionalen wie internationalen Institutionen (vgl. WYBIEREK et al. 2019), so dass die Hochschullernwerkstatt eine bekannte Größe im regionalen Bildungsraum wird. 


\section{Arbeitsschwerpunkte und Einflussfaktoren}

Aus den obigen Betrachtungen lassen sich Einflüsse ableiten, die auf die Einrichtung (Phase I) bzw. die inhaltliche Ausgestaltung, Erweiterung oder Neuorientierung (Phase 3, 5) von Hochschullernwerkstätten einwirken (a). Weiterhin zeichnen sich Kontexte ab, in denen die in oder durch Hochschullernwerkstätten initiierten Aktivitäten auch auf Strukturen, Kulturen, Haltungen oder Praktiken in anderen Bereichen der Hochschule einwirken (b). Zuletzt, und in einem reflexiven Prozess bedeutsam, sind Einflussfaktoren, die eher auf interne Entwicklungsprozesse wirken wie Team-, Konzept- und Organisationsentwicklung, ggf. auch Lehrentwicklung (adaptiert vgl. RolfF 2016) (c).

a) Durch professorale Initiativen und finanzielle Unterstützung wird die Etablierung von Hochschullernwerkstätten erleichtert.

Inhaltlich wird das Geschehen in der halleschen Hochschullernwerkstatt stark durch aktuelle gesellschaftliche Themen (Nachhaltigkeit, sharing community, Feminismus, Datenschutz und Digitalisierung, Rechtsextremismus, Fluchtbewegungen, Kommunikationsformen, menschenrechtsbasierte Bildung u.v.a.m.) oder Interessen Einzelner (Handwerk, Zirkuspädagogik etc.) beeinflusst, da Studierende oder Mitarbeiter*innen diese Themen einbringen.

Die Studienstrukturen und die zeitliche Eingebundenheit der Studierenden in Verbindung mit einer (geringen) curricularen Einbindung kann die Etablierung der Hochschullernwerkstatt als feste Größe und so die Breitenwirkung sicher beeinflussen. Unserer Erfahrung nach scheint dies aber weniger bedeutsam als transparente und offene Möglichkeiten studentischer Beteiligung.

Dagegen zeigen die Entwicklungen, dass zum einen die Bereitstellung von Personalstellen nötig ist, die Kontinuität und längerfristige Kooperationen ermöglichen und den umfangreichen Verwaltungsaufgaben nachkommen können. Zum anderen, dass das Vorhandensein von Qualifikationsstellen Grundvoraussetzung für eine umfangreich wahrgenommene Hochschullernwerkstatt ist, die konzeptionelle Entwicklungen und die notwendige Forschung in diesem Feld zulässt. Dies verdeutlicht u. a. kontrastierend zu Phase II (2.2) die Entwicklung der Hochschullernwerkstatt in Erfurt (vgl. Godau et al. 2019). Das Vorhandensein zuständiger Personen in Verbindung mit finanziellen Ressourcen setzt starke Entwicklungsimpulse. Die Verfügbarkeit zentraler und gemütlicher Räume ist für die Veränderung hochschulischen Lebens und informeller Praktiken zudem maßgeblich. Ob die Einbindung in Hochschulstrukturen über professorale Verantwortlichkeit - wie in den Gründungsjahren in Halle - oder über stärker partizipatorisch gedachte Strukturen (möglicherweise in Kollektiv-Form?) günstiger ist, bleibt zu erproben.

Bestehende Netzwerke in der Universität (z. B. der Lehrer*innenbildung, studentische Netzwerke) und der Region und deren Nutzung können die Sicht- 
barkeit, Anerkennung und die kooperative Angebotsentwicklung verbessern, bewirken aber auch einen erhöhten Organisationsaufwand.

b) Das Vorhandensein einer Hochschullernwerkstatt erfordert die Auseinandersetzung mit dieser Institution und ihren konzeptionellen Ansätzen (vgl. 2.2). Die Veranstaltung von Tagungen (am eigenen Standort) sowie die Vernetzung und Publikation im Rahmen des Netzwerkes NeHle tragen zur Sichtbarkeit sowie zur Weitergabe inhaltlicher Impulse (2.5), aber auch zur Festigung von Kommunikationsstrukturen entscheidend bei. Indem sie Gelegenheiten schafft (z. B. Materialitätsforum, Hochschuldidaktische Austauschrunden) und Räume für Kooperationen bereitstellt, wirkt die Hochschullernwerkstatt vernetzend auf die Universität zurück. Über Lehrkooperationen, hochschuldidaktischen Austausch oder studentische Initiativen mit Lernwerkstatterfahrung wurden einzelne Lernformen verändert. Dafür sind gelingende Austauschformate nötig.

Einwirkungen auf Lernkultur und Haltungen der Pädagog*innen in Schulen und kooperierenden Institutionen sind annehmbar, da Beobachtungsmöglichkeiten und einzelfallbezogene gemeinsame Reflexionen mit Studierenden und Pädagog*innen in den Projekten Anregung bieten. Studierende als zukünftige Lehrende und Multiplikator*innen (gleich, ob in Lehrveranstaltungen, Projekten oder frei in der Hochschullernwerkstatt aktiv) lassen sensible und differenzierte Sichtweisen auf heterogene Lernvoraussetzungen und Lernprozessgestaltung erkennen (Sснӧрs 2019; RumpF \& Sснӧрs 2017). Inwiefern diese aber länger wirken und Erfahrungen bspw. der 2. Phase überdauern, ist bisher nicht belegt (vgl. Godau et al. 2018, 48).

Gelungen ist der Zugang für zwei Personen ohne eigentliche Hochschulzugangsberechtigung und damit die Entwicklung einer inklusiveren Umgebung (vgl. K. Kramer in diesem Band), Voraussetzung dafür war hohes persönliches Engagement.

c) Die Professionalisierungsverständnisse der Gestaltenden nehmen deutlich Einfluss auf die Ausrichtung der Lehrangebote oder des gesamten konzeptionellen Rahmens.

Für die Beteiligung Dozierender und die gemeinsame Lehr-Entwicklung bedarf es gut entwickelter Strukturen für die Zusammenarbeit und gemeinsames Zuständigkeitserleben - insbesondere für interessierte Lehrende aus anderen universitären Bereichen.

Ausgehend von der gezeigten Entwicklung in Halle kann man schließen: Hochschullernwerkstätten sind Kontexte, die es - eine flexible Gestaltung und interessensorientierte Offenheit vorausgesetzt - leicht ermöglichen, inhaltlich aktuelle gesellschaftliche Diskurse in Zusammenhänge der pädagogischen Bildung $\mathrm{zu}$ integrieren und soziale Interaktionsstrukturen zu explorieren. Hier stehen sie vielleicht in Opposition zu curricularen Lehrveranstaltungen, die in der Hoch- 
schulstruktur nur einer gewissen inhaltlichen Offenheit folgen können und durch wenige Personen verantwortet sind. Hochschullernwerkstätten können daher sowohl für Entwicklungen der gesamten Hochschule (Erprobungen struktureller Veränderungen, vgl. 2.5 und Kramer, K. in diesem Band) oder einzelne Bereiche der Pädagog*innenbildung (hochschuldidaktische Veränderungen oder partizipatorische Entwicklungsansätze, vgl. Godau et al. 2018) als auch für einzelne Studierende und Dozierende einen Experimentalraum bilden.

\section{Fazit}

Die in den Anfängen der Hochschullernwerkstätten im Raum Halle bereits konzeptuell verankerten Ansprüche an eine Professionalisierung hinsichtlich der Befähigung zum Umgang mit veränderten schulischen und gesellschaftlichen Bedingungen (Schelenz 1993) sowie das Anstreben von veränderten Haltungen und Lernkulturen (Kunze \& MEYer 1996) durch Selbsterfahrung wurden in den späteren Phasen aufgegriffen. Das Bestreben, Innovation von Schul- und Unterrichtsgestaltung voranzutreiben - in direkter Kooperation mit Institutionen ,der Praxis' ebenso wie in hochschulischen Bildungsangeboten - scheint durchgängige Motivation der Einrichtung solcher Lernorte zu sein. Auch der Wunsch, diese Orte nicht aus einer Fachperspektive zu ,bespielen', sondern in gemeinsamer Verantwortung übergreifend zu gestalten, ist durchgängig vorhanden, scheint sich aber einer umfassenden Realisierung immer wieder zu entziehen.

Während die gesamtgesellschaftlichen Entwicklungen und Debatten der letzten Dekaden anschlussfähig an Hochschullernwerkstattkonzeptionen waren und von Lernwerkstatt-Gestalter*innen gut für die konzeptionelle Entwicklung genutzt werden konnten, sind Ausstrahlungen der Entwicklungen der Hochschullernwerkstatt in Halle in andere universitäre Bereiche und Strukturen verstärkt wahrnehmbar. Zunehmend ist „unsere“ Hochschullernwerkstatt universitätsweit bekannt und wird als innovativer Lernort gern präsentiert. Die Frage, inwiefern eine solche ,Institution' in die komplexe pädagogische Realität einer Universität bzw. Hochschule hineinwirken kann, ist Gegenstand wiederkehrender teaminterner Reflexionen. Dieser Frage hoffen wir mit diesem Band aus einer Gesamtperspektive näher zu kommen.

\section{Literatur}

Asmus, Miriam; Friebe, Kati; Lewin Mirjam \& Misselwitz, Kati (2020): Entdeckendes Lernen und Digitale Medien - ein Traumpaar. In diesem Band.

Beyer, Constantin \& Johnke-Liese, Florian (2020): Hochschullernwerkstatt goes digital! In diesem Band. 
Воотн, Tony \& Ainscow, Mel (2011): index for inclusion. developing learning and participation in schools. Csie. Online unter: http://prsinstitute.org/downloads/related/education/IndexforInclusion.pdf.

Breidenstein, Georg; Burkhardt, Sara; Rabe, Thorid \& Schöps, Miriam (2020): Zur Materialität des Lernens Anregungen aus einem interdisziplinären Forum in der Hochschullernwerkstatt. In diesem Band.

Godau, Marc; Tänzer, Sandra; Berger, Marcus; Lingemann, Jana \& Mannhaupt, Gerd (2018): Studieren in der Lernwerkstatt. Erwartungen, Möglichkeiten und Herausforderungen für die Lehrer/innenbildung (nicht nur) in der Didaktik des Sachunterrichts. In: GDSU Journal, Heft 8, 47-65. Online unter: http://www.gdsu.de/gdsu/wp-content/uploads/2019/02/47_65_taenzer.pdf.

Godau, Marc; Tänzer, Sandra; Berger, Marcus; Mannhaupt, Gerd \& Knigge, Jens (2019): Implementation einer Hochschullernwerkstatt. Zur Entwicklung des Lernwerkstatt-Konzepts an der Universität Erfurt. In: BaAr, Robert; Feindt, Andres \& Trostmann, Sven (Hrsg.): Struktur und Handlung in Lernwerkstätten. Hochschuldidaktische Räume zwischen Einschränkung und Ermöglichung. Bad Heilbrunn: Klinkhardt, 120-132.

Grummt, Marek; Schöps, Miriam \& Veber, Marcel (2019a): Der Raum als 3. Pädagog*in in der kasuistischen Lehrer*innenbildung. In: BaAR, Robert; Feindt, Andreas \& Trostmann, Sven (Hrsg.): Struktur und Handlung in Lernwerkstätten - Hochschuldidaktische Räume zwischen Einschränkung und Ermöglichung. Bad Heilbrunn: Klinkhardt, 229-223.

Grummt, Marek; Schöps, Miriam \& Veber, Marcel (2019b): Synergien kasuistischer Forschungswerkstatt und Lernwerkstatt - Reflexionsanregung durch räumlich bereicherte Forschungsarbeit. In: Tänzer, Sandra; Mannhaupt, Gerd; Berger, Marcus \& Godau, Marc (Hrsg.): Perspektiven auf Hochschullernwerkstätten. Wechselspiele zwischen Individuum, Gemeinschaft, Ding und Raum. Bad Heilbrunn: Klinkhardt, 57-69.

Hericks Uwe; Kunze, Ingrid \& Trautmann, Matthias (2019): Nachruf auf Prof. Dr. Meinert Meyer. Erziehungswissenschaft Heft 58, Jg. 30, 141-143.

Hochschullernwerkstatt Erziehungswissenschaften (HSLW) (2018): Digitaler Lern- und Forschungsraum. Online unter: https://www.philfak3.uni-halle.de/institut/ hochschullernwerkstatt/dielernwerkstatt/2640678_2885615/.

Karge, Sabine \& HüBner, Riccarda (2015): Erinnerungen an die Entstehung der Lernwerkstatt Aufzeichnung eines Gesprächs vom 27.02.2015.

KLAFKI, Wolfgang \& BraUn, Karl-Heinz (2007): Wege pädagogischen Denkens. Ein autobiografischer und erziehungswissenschaftlicher Dialog. München: Reinhardt.

Kramer, Kathrin (2020): Nichtreformistische Reformen - die Bedeutung von Hochschullernwerkstätten auf dem Weg zu einer inklusiven Universität am Beispiel des Budgets für Arbeit. In diesem Band.

Kramer, Kathrin; Rumpf, Dietlinde; Schöps, Miriam \& Spuller, Siglinde (2019): Die Ambivalenz strukturierender Bedingungen. Überlegungen zu Einflussfaktoren auf studentische Tätigkeiten in der Lernwerkstatt. In: BaAR, Robert; Trostmann, Sven \& Feindt, Andreas (Hrsg.): Struktur und Handlung in Lernwerkstätten - Hochschuldidaktische Räume zwischen Einschränkung und Ermöglichung. Bad Heilbrunn: Klinkhardt, 133-144.

Kramer, Rolf Torsten (2020): Zum Problem der Professionalisierung im Lehramtsstudium und zum Potential der Hochschullernwerkstatt. In diesem Band.

KunZe, Ingrid \& Meyer, Meinert (1996 unveröff.): Interview mit Frau Dr. I. Kunze und Herrn Prof. Dr. M. Meyer zur Lernwerkstatt in Halle. Manuskript/Transkript, Halle.

LeRnWERKSTATt ERZiehungSWisSENSChaften (2009) (LW Ew): Systematisierung Lernwerkstatt. Internes Dokument zur Materialsortierung und Regalbezeichnung, o.A., unveröffentlicht - Stand Mai 2009, Halle. 


\section{Miriam Schöps und Dietlinde Rumpf}

Lernwerkstatt Erziehungswissenschaften (LW Ew) (Hg.) (2015): Was ist die Lernwerkstatt? Selbstverständnis. MLU Halle. (Autorin: Miriam Schöps). Online unter: https://www.philfak3. uni-halle.de/institut/hochschullernwerkstatt/dielernwerkstatt/2640678_2885615/.

Lewex, Tobias (2019): Das Außerunterrichtliche Pädagogische Praktikum - Heterogene Erfahrungsräume im Spannungsfeld von Theorie und Praxis. In: Klektau, Claudia; Schütz, Susanne \& FETT, Anne Julia (Hrsg.): Heterogenitätssensibilität durch Fallarbeit fördern. Zum Stellenwert von Kasuistik und Inklusion in der Lehrer*innenbidung. MLU Halle. 67-76.

RolfF, Hans-Günter (2016): Schulentwicklung kompakt. Modelle, Instrumente, Perspektiven. Weinheim, Basel: Beltz (Pädagogik).

Rumpf, Dietlinde \& Schöps, Miriam (2013): Hochschulwerkstätten als Raum für Kooperation. In: Coelen, Hendrik \& Müller-Naendrup, Barbara (Hrsg.): Studieren in Lernwerkstätten. Potentiale und Herausforderungen für die Lehrerbildung, Wiesbaden: Springer VS, 31-39.

Rumpf, Dietlinde \& Schöps, Miriam (2017): Reflexion als Gegenstand qualitativer Forschung in der Arbeit der Lernwerkstatt Erziehungswissenschaften/Halle. In: Kekeritz, Mirja; Graf, Ulrike; Brenne, Andreas; Fiegert, Monika; Gläser, Eva \& Kunze, Ingrid (Hrsg.): Lernwerkstattarbeit als Prinzip. Möglichkeiten für Lehre und Forschung. Bad Heilbrunn: Klinkhardt, 85-99.

Schelenz, Ute (1993): Zu den Aufgaben der Lernwerkstätten an der Hochschule. „Wolfgang Ratke“-Institut, Köthen (unveröffentlichtes Manuskript).

ScHLAG, Melanie (2020): Hochschullernwerkstatt schlägt Schlager. In diesem Band.

Sснӧрs, Miriam (2019): „Also ganz homogen warn die irgendwie nich“- Auseinandersetzungen mit Vielfalt in der Hochschullernwerkstatt. In: Klektau, Claudia; Schütz, Susanne \& Fetт, Anne Julia (Hrsg.): Heterogenitätssensibilität durch Fallarbeit fördern. Zum Stellenwert von Kasuistik und Inklusion in der Lehrer*innenbidung. MLU Halle, 107-114.

Schöps, Miriam (2017): Teilprojekt Ausbau der Hochschullernwerkstatt. KALEI-Projekt MLU Halle. Online unter: https://kalei.uni-halle.de/kalei1/teilprojekte/ausbau_der_lernwerkstatt/.

Schöps, Miriam (2016): Inklusive Praxis in Hochschullernwerkstätten. In: Boban, Ines \& Hinz, Andreas (Hrsg.): Arbeit mit dem Index für Inklusion. Entwicklungen in weiterführenden Schulen. Bad Heilbrunn: Klinkhardt.

Schöps, Miriam (2015): Schulkooperation Gym - Projektgebundene Begleitung von themenbezogenen Lern- und Entwicklungsprozessen. Online unter: https://www.philfak3.uni-halle.de/institut/hochschullernwerkstatt/1050437_2967008/2967008_2967014/(Abrufdatum: 15.06.2020).

Sснӧрs, Miriam (2012): Lernwerkstatt zwischen Angebot und Nachfrage? Lernwerkstatt Erziehungswissenschaften als Ort für Studienkultur! Poster zur 5. Fachtagung der Hochschullernwerkstätten „Studieren in Lernwerkstätten“; Siegen, 12.-14. Februar 2012.

Sснӧps, Miriam \& RumpF, Dietlinde (2015): 10 Jahre Lernwerkstatt an der Universität Halle - den Kinderschuhen entwachsen? Von der belächelten Praxisspielwiese auf dem Weg zum Forschungsprojekt. Vortrag zur Jubiläumstagung „Lernwerkstätten und die Professionalität von Lehrer*innen in einer zukünftigen Schule“, 10.6.2015, Halle.

WENZEL, Hartmut (2020): Lernwerkstätten auch für die Sekundarstufen - Hintergründe für die Entwicklung der Hochschullernwerkstatt in Halle. In diesem Band.

WybiereK, Kira; Kramer, Kathrin \& Schöps, Miriam: Aus Kooperationen werden Kollaborationen. Die Lernwerkstatt Erziehungswissenschaften Halle im Netzwerk (Poster) Brixen, 14.2.2019. 